\title{
Related-Key Attack on Full-Round PICARO *
}

\author{
Anne Canteaut, Virginie Lallemand and María Naya-Plasencia \\ Inria, project-team SECRET, France \\ \{Anne.Canteaut, Virginie.Lallemand, Maria.Naya_Plasencia\}@inria.fr
}

\begin{abstract}
Side-channel cryptanalysis is a very efficient class of attacks that recovers secret information by exploiting the physical leakage of a device executing a cryptographic computation. To adress this type of attack, many countermeasures have been proposed, and some papers adressed the question of constructing an efficient masking scheme for existing ciphers. In their work, G. Piret, T. Roche and C. Carlet took the problem the other way around and specifically designed a cipher that would be easy to mask. Their careful analysis, that started with the design of an adapted Sbox, leads to the construction of a 12-round Feistel cipher named PICARO. In this paper, we present the first fullround cryptanalysis of this cipher and show how to recover the key in the related-key model. Our analysis takes advantage of the low diffusion of the key schedule together with the non-bijectivity of PICARO Sbox. Our best trade-off has a time complexity equivalent to $2^{107.4}$ encryptions, a data complexity of $2^{99}$ plaintexts and requires to store $2^{17}$ (plaintext, ciphertext) pairs.
\end{abstract}

Keywords: related-key attack, differential cryptanalysis, PICARO.

\section{Introduction}

While performance and side-channel attacks resistance are most of the time considered separately and as distinct problems - new design papers focus on performance figures while countermeasure papers focus on how to implement specific protection in order to reduce performance overheads - , some new cipher proposals tackle the two problems together by designing new primitives that fit given protections. Examples of such constructions are PICARO [8], Zorro [], and the family of LS-design [7] (including Robin and Fantomas as concrete instantiations).

The countermeasure studied in these three designs is the masking scheme 10] for which the heavier parts to protect are the operations which are not linear with respect to the group operation used to share the sensitive variables. For these three ciphers as for most ciphers, these non-linear operations are concentrated in the Sboxes, and then the straightforward way to limit the masking cost is to

*Partially supported by the French Agence Nationale de la Recherche through the BLOC project under Contract ANR-11-INS-011. 
reduce the number of Sbox application $\mathrm{S}^{1}$ as well as to choose Sboxes that are masking-friendly, for instance by reducing the number of field multiplications processed 2 . This later direction is the one followed by Piret, Roche and Carlet while devising PICARO [89]: their design relies on a non-bijective Sbox defined by two bivariate polynomials over $G F\left(2^{4}\right)$, reducing the number of non-linear multiplications to four. This Sbox is integrated to the round function of a Feistel scheme, composed of four operations which are an expansion, a key addition, an Sbox-layer and a compression.

In this paper we show that despite the fact that the authors of PICARO aim at resisting to related-key attacks, as pointed out in $[9]^{3}$ we have been able to mount related-key attacks on the full cipher. Our attacks exploit some weaknesses in the key-schedule, the non-bijective properties of the Sbox and some properties of the linear layer. They provide different trade-offs between time and data complexities.

The paper is organized as follows. In Section 2, we give a brief description of the PICARO block cipher and of its design choices, in Section 3 we give some definitions and in Section 4. we present an analysis of PICARO key-schedule that leads to the related-key attack described in Section 5 . The paper ends with a conclusion.

\section{Description of PICARO}

\subsection{Round Function}

One of the main objectives of G. Piret, T. Roche and C. Carlet when designing PICARO was to propose a 128-bit block cipher that would get an advantage over other ciphers regarding the ease to protect against side-channel attacks. To achieve this, they started from Rivain and Prouff's masking scheme [10] and determined which operations are difficult to mask to derive some new design criteria. Their analysis brought to light that efforts have to focus on the Sboxes since the masking scheme implies heavy overheads for the non-linear operations.

This condition must be added to the usual criteria coming from the (nonphysical) usual attacks (including the prominent linear, differential, algebraic cryptanalyses and their variants) that require the Sbox be highly non-linear, have a high algebraic degree and a low differential-uniformity.

Their deep analysis resulted in the selection of an Sbox defined as the concatenation of two polynomials:

$$
\begin{aligned}
S: G F\left(2^{4}\right) \times G F\left(2^{4}\right) & \rightarrow G F\left(2^{4}\right) \times G F\left(2^{4}\right) \\
(x, y) & \mapsto\left(x y,\left(x^{3}+0 \times 02\right)\left(y^{3}+0 \times 04\right)\right)
\end{aligned}
$$

\footnotetext{
${ }^{1}$ This direction has been followed in the design of Zorro.

${ }^{2}$ When considering binary masking, this criterion is equivalent to limiting the number of AND processed (see for instance the LS-design [7]).

${ }^{3}$ Section 7.2 of [9]: "We want our scheme to resist known attacks on a key schedule algorithm, in particular related-key attacks..."
} 
where $0 \mathrm{x} 02$ and $0 \mathrm{x} 04$ represent elements of $G F\left(2^{4}\right)$ defined as $G F(2)[x] /\left(X^{4}+\right.$ $\left.X^{3}+1\right)$. Its full look-up table is given in Appendix A.

This non-bijective Sbox has already been studied in [5] and possesses the following desirable characteristics: a non-linearity equal to 94 , an algebraic degree equal to 4 , a maximal differential probability equal to $4 / 256$. Furthermore, to fit well the masking protection, it can be implemented with only 4 non-linear operations; moreover all these non-linear operations are defined in the small field $G F\left(2^{4}\right)$.

An obvious but quite important remark that has to be made is that since the Sbox is not bijective, there exist sets of values that all have the same image through the Sbox. In other words, it is possible to cancel a difference entering the Sbox: a byte that is active before applying the Sbox can lead to an inactive output. Since the cube function is 3-to- 1 over $G F\left(2^{4}\right)^{*}$, we deduce from the definition of the Sbox that it is a 3-to-1 function over $G F\left(2^{4}\right)^{2} \backslash\{(0,0)\}$. Moreover, it is very easy to prove that, for any non-zero input difference $\Delta$, the transition $\Delta \rightarrow 0$ holds with probability $2^{-7}$. In other words, the equation $S(x+\Delta)+S(x)=0$ has exactly two solutions $x$.

The choice of a non-bijective Sbox was motivated by the fact that finding a good Sbox is easier in this case. However, it requires to find a way to include it in a construction that makes the cipher invertible, and also to take the resulting differential properties into account. Therefore, this non-bijective Sbox is used within the Feistel construction. But, as noticed by the designers, the use of a basic Sbox layer as an inner function would make the cipher vulnerable to a quite simple but very efficient differential attack with only one active Sbox every 2 rounds.

To thwart this sort of attacks, PICARO designers choose to use an expansion and a compression function that have good diffusion, more precisely that ensure that a minimum of 7 Sboxes are active in each active round. This is achieved by using linear operations deduced from linear codes with minimum distance 7 . The expansion $G$ takes as input a 64 -bit word and outputs an extended word of 112 bits. The corresponding matrix is depicted below (its entries are elements in $G F\left(2^{8}\right)$ defined as $\left.G F(2)[X] /\left(1+X^{2}+X^{3}+X^{4}+X^{8}\right)\right)$. Then, the inner state must be compressed back at the end of the round function. To this end, another linear operation is used, defined by the matrix $H=G^{T}$, which converts 112-bit words into 64-bit ones.

$$
G=\left(\begin{array}{llllllllllllll}
01 & 00 & 00 & 00 & 00 & 00 & 00 & 00 & 01 & 01 & 0 A & 01 & 09 & 0 C \\
00 & 01 & 00 & 00 & 00 & 00 & 00 & 00 & 05 & 01 & 01 & 0 A & 01 & 09 \\
00 & 00 & 01 & 00 & 00 & 00 & 00 & 00 & 06 & 05 & 01 & 01 & 0 A & 01 \\
00 & 00 & 00 & 01 & 00 & 00 & 00 & 00 & 0 C & 06 & 05 & 01 & 01 & 0 A \\
00 & 00 & 00 & 00 & 01 & 00 & 00 & 00 & 09 & 0 C & 06 & 05 & 01 & 01 \\
00 & 00 & 00 & 00 & 00 & 01 & 00 & 00 & 01 & 09 & 0 C & 06 & 05 & 01 \\
00 & 00 & 00 & 00 & 00 & 00 & 01 & 00 & 0 A & 01 & 09 & 0 C & 06 & 05 \\
00 & 00 & 00 & 00 & 00 & 00 & 00 & 01 & 01 & 0 A & 01 & 09 & 0 C & 06
\end{array}\right)
$$


To sum up, the round function is made of four operations, as depicted on Figure 1. first, the 64-bit left part is extended by the expansion function defined by $G$, then the round-key is added. This is followed by the Sbox application (14 parallel applications of $S$ ) and finally the state is compressed back to 64 bits, and is XORed to the right part of the internal state.

PICARO encryption routine is made of 12 iterations of this round function.

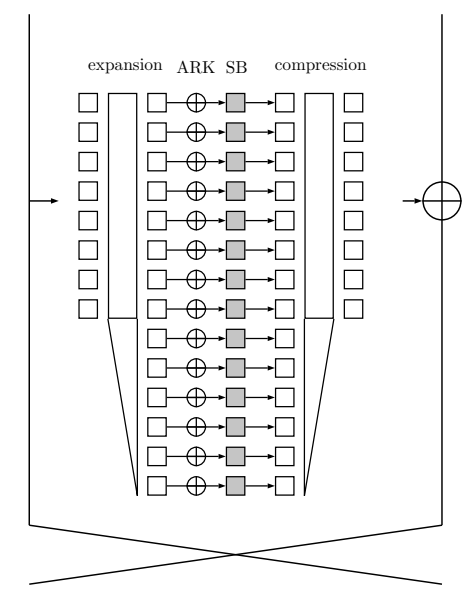

Fig. 1. One round of PICARO block cipher.

\subsection{Key-Schedule}

The previous algorithm requires twelve 112-bit subkeys that are derived from the 128-bit master key $K$. The designers wanted a simple and efficient key-schedule, together with resistance to the two main attacks exploiting the key-schedule, namely related-key attacks 1 and slide attacks [3. In addition to that, Piret et al. looked for round-keys that could be computed on the fly, i.e. for which the computation of the subkey of round $i$ can be done from the knowledge of the subkey at round $(i-1)$ (or from the knowledge of the subkey at round $(i+1)$, in decryption mode).

Their analysis has resulted in a linear key-schedule composed of rotations, bitwise additions and truncations. Namely, $K$ denotes the 128-bit master key and $K^{(1)}, K^{(2)}, K^{(3)}$ and $K^{(4)}$ are the four 32-bit chunks composing $K: K=$ $\left(K^{(1)}, K^{(2)}, K^{(3)}, K^{(4)}\right)$. Let $T:\left(G F(2)^{32}\right)^{4} \rightarrow\left(G F(2)^{32}\right)^{4}$ be the linear transformation defined as follows:

$$
\left(\begin{array}{l}
T(K)^{(1)} \\
T(K)^{(2)} \\
T(K)^{(3)} \\
T(K)^{(4)}
\end{array}\right)=\left(\begin{array}{llll}
0 & 1 & 1 & 1 \\
1 & 0 & 1 & 1 \\
1 & 1 & 0 & 1 \\
1 & 1 & 1 & 0
\end{array}\right) \times\left(\begin{array}{l}
K^{(1)} \\
K^{(2)} \\
K^{(3)} \\
K^{(4)}
\end{array}\right)
$$


Then, to compute the subkeys from the master key $K$, we first compute the extended key $\left(\kappa^{1}, \kappa^{2}, \cdots \kappa^{12}\right)$ (where each $\kappa^{i}$ is 128-bit long) with the following formulas:

$$
\begin{cases}\kappa^{1}=K & \\ \kappa^{i}=T(K) \ggg \Theta(i) & \text { for } i=2,4,6,8,10,12 \\ \kappa^{i}=K \ggg \Theta(i) & \text { for } i=3,5,7,9,11\end{cases}
$$

where $\Theta(i)$ is:

\begin{tabular}{c|ccccccccccc}
$\mathrm{i}$ & 2 & 3 & 4 & 5 & 6 & 7 & 8 & 9 & 10 & 11 & 12 \\
\hline$\Theta(i)$ & 1 & 16 & 17 & 32 & 33 & 85 & 86 & 101 & 102 & 117 & 118
\end{tabular}

and then we obtain the round keys $k^{i}$ by extracting the first 112 bits from $\kappa^{i}$. We denote by $s k w=\left(k^{1}\left\|k^{2}\right\| \ldots k^{12}\right)$ the 'subkey word' made of the concatenation of the $k^{i}$.

The fact that $T$ is an involution allows to easily deduce the 'on-the-fly' expressions, which are:

$$
\left\{\begin{array}{l}
\kappa^{1}=K \\
\kappa^{i}=T\left(\kappa^{i-1}\right) \ggg \theta(i) \quad \text { for } i=2, \cdots, 12
\end{array}\right.
$$

where $\theta$ is defined by:

\begin{tabular}{c|ccccccccccc}
$\mathrm{i}$ & 2 & 3 & 4 & 5 & 6 & 7 & 8 & 9 & 10 & 11 & 12 \\
\hline$\theta(i)$ & 1 & 15 & 1 & 15 & 1 & 52 & 1 & 15 & 1 & 15 & 1
\end{tabular}

Since we do not need it here, we refer to the design document 8 for the formulas of the round-key derivation in decryption mode.

\section{Definitions and Notation}

In most block cipher constructions, the Sboxes are bijective, implying that in a differential attack a non-zero difference at the input of an Sbox is equivalent to a non-zero difference at its output. Here, the situation is different since the Sboxes are not bijective. Therefore, in order to avoid any ambiguity, we give a more precise definition of the notion of active Sbox:

Definition 1 (Active Sbox). An active Sbox is an Sbox with a nonzero input difference (and a possibly zero output difference).

\section{Definition 2 (Data, Time and Memory Complexities).}

- The data complexity is defined as the number of plaintext/ciphertext pairs necessary to conduct the attack;

- The time complexity, which is expressed as a number of full 12-round encryptions, incorporates all the operations performed by the attacker to recover the $k e y$, and includes the encryptions needed to compute the necessary data; 
- The memory complexity, which is expressed as a number of 128-bit blocks, measures the memory needed during the attack.

Definition 3 ( $n \mathbf{R}$-attack [2]). A nR-attack is a differential attack that covers $R$ rounds with a differential characteristic and attacks $R+n$ rounds in total. We extend this definition to the related-key setting and call the additional rounds the key-recovery rounds.

\section{Key-Schedule Analysis}

In this section we focus on PICARO key-schedule. We first focus on keys under which a given plaintext leads to the same ciphertext and then extend our analysis to a related-key attack.

\subsection{Keys Leading to Colliding Ciphertexts}

In this section we are interested in sets of keys that with high probability encrypt a given plaintext into the same ciphertext after the 12 rounds of PICARO. Note that in the ideal case, if one plaintext is fixed and encrypted under different keys, assuming that the resulting function is random, a ciphertext collision is expected to occur with probability $2^{-128}$.

In the case of PICARO, we can remark that the round structure enables us to cancel a key difference quite easily: indeed, since the key addition is immediately followed by the Sbox layer, composed of non-bijective Sboxes, a key difference can be immediately canceled by going through the Sbox, resulting in an internalstate collision at the input of the compression function.

Obtaining colliding ciphertexts becomes then possible if we can construct keys differing in as few positions as possible, in order to make the probability of the event "all the subkey differences are canceled by the Sboxes" higher than $2^{-128}$. This means that we can cancel a maximum of $s$ Sboxes, with $s$ satisfying $2^{-7 \times s}>2^{-128}$, so we have to find keys such that the corresponding subkey words differ in at most 18 bytes.

To find such keys, we first remark that the key-schedule algorithm is linear over $G F(2)$. This implies that the words made by the concatenation of the subkeys (skw) belong to a linear code $C$ of dimension $k=128$ and length $n=112 \times 12=1344$. Our search then boils down to the search for codewords with a low Hamming weight. We first focus on the Hamming weights of the codewords over $G F(2)$ and we will then move to the Hamming weight over $G F\left(2^{8}\right)$, i.e., the number of non-zero bytes, which is the relevant parameter in our attack.

To determine if it is possible to obtain keys differing in less than 18 bytes, i.e, at the inputs of less than 18 Sboxes, a first idea is to compute the minimum distance of the binary code, hereafter denoted by $d$. A straightforward computation of $d$ would be too complicated due to the large size of the code. Some algorithms for finding low-weight codewords, e.g [4, could be used to determine $d$. But we 
now show that it can be easily deduced from the structure of the code with very simple arguments. We first make some observations coming from the structure of the generator matrix depicted on Figure 2.

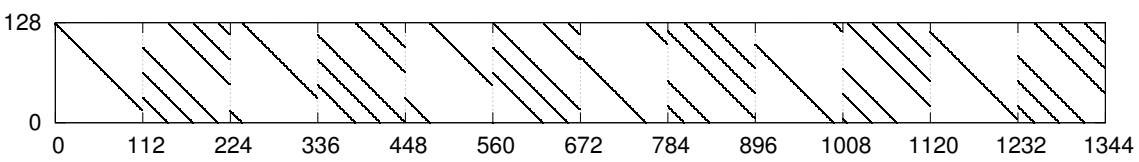

Fig. 2. Graphical representation of the generator matrix of linear code corresponding to the key-schedule.

- If we consider all possible master keys of weight 1 , the minimum weight among all corresponding codewords is 20, implying that $d \leq 20$

- According to the key-schedule description, 6 subkeys, namely $k^{1}, k^{3}, k^{5}, k^{7}$, $k^{9}$ and $k^{11}$ consist of a selection of bits from the master key $K$. Following this, if we consider a master key of weight 1 , then the word made by the concatenation of the subkeys skw contains at least 4 ones. Indeed, we have a 1 for sure in $\kappa^{1}, \kappa^{3}, \kappa^{5}, \kappa^{7}, \kappa^{9}$ and $\kappa^{11}$ but after the truncation, a maximum of two of these ones may disappear. Accordingly, every time we add a one in the master key, the weights of the odd subkey words increase by at least 4 .

All in one, those remarks show that, if they exist, the codewords of weight strictly less than 20 are obtained from master keys of weight at most 4 . An exhaustive search over all these master keys shows that the minimum distance of the code is $d=18$. The active positions of all master keys that reach this minimum are given in Table 1 .

Table 1. Positions of the active bits in the round-keys that correspond to a minimumweight subkey word (the bits entering the first Sbox are indexed from 0 to 7 ).

\begin{tabular}{|c||c||c|c|c|c|c|c|c|c|c|c|c|c|}
\hline set & $K$ & $k^{1}$ & $k^{2}$ & $k^{3}$ & $k^{4}$ & $k^{5}$ & $k^{6}$ & $k^{7}$ & $k^{8}$ & $k^{9}$ & $k^{10}$ & $k^{11}$ & $k^{12}$ \\
\hline \hline 1 & 27,123 & 27 & 28 & 11,43 & 12,44 & 27,59 & 28,60 & 80 & 81 & 0,96 & 1,97 & 16 & 17 \\
\hline 2 & 28,124 & 28 & 29 & 12,44 & 13,45 & 28,60 & 29,61 & 81 & 82 & 1,97 & 2,98 & 17 & 18 \\
\hline 3 & 29,125 & 29 & 30 & 13,45 & 14,46 & 29,61 & 30,62 & 82 & 83 & 2,98 & 3,99 & 18 & 19 \\
\hline 4 & 30,126 & 30 & 31 & 14,46 & 15,47 & 30,62 & 31,63 & 83 & 84 & 3,99 & 4,100 & 19 & 20 \\
\hline \hline 5 & 91,123 & 91 & 92 & 11,107 & 12,108 & 27 & 28 & 48,80 & 49,81 & 64,96 & 65,97 & 80 & 81 \\
\hline 6 & 92,124 & 92 & 93 & 12,108 & 13,109 & 28 & 29 & 49,81 & 50,82 & 65,97 & 66,98 & 81 & 82 \\
\hline 7 & 93,125 & 93 & 94 & 13,109 & 14,110 & 29 & 30 & 50,82 & 51,83 & 66,98 & 67,99 & 82 & 83 \\
\hline 8 & 94,126 & 94 & 95 & 14,110 & 15,111 & 30 & 31 & 51,83 & 52,84 & 67,99 & 68,100 & 83 & 84 \\
\hline
\end{tabular}


Note that these configurations might have been expected: the best scenarios are the ones for which the differences do not propagate a lot, so for keys differing in more than 2 bits we are looking for differences that cancel each other by the $T$ map, i.e. that are at the same relative position in the 32-bit chunks of the master key. Moreover, the best configurations correspond to differences that end up as often as possible in the truncated part of the subkeys.

We note that the previous analysis determines the subkey words having a low Hamming weight counted in bits, while the relevant quantity is the number of nonzero bytes since it corresponds to the number of active Sboxes. However, the structure of the previous result indicates that the words with minimal weight over $G F\left(2^{8}\right)$ are derived from master keys having at most two nonzero bytes. An exhaustive search over this set enables us to determine a set $W$ of 30 minimumweight codewords over $G F\left(2^{8}\right)$, corresponding to the $\left(2^{4}-1\right)$ non-zero linear combinations of the first four rows in Table 1 and to the $\left(2^{4}-1\right)$ non-zero linear combinations of the last four rows. This analysis exhibits the following bias in PICARO:

The probability that any given plaintext is encrypted to the same ciphertext under two keys whose difference belongs to the set $W$ is $\left(2^{-7}\right)^{18}=2^{-126}$.

\section{Related-Key Attack on the Full-Round PICARO}

We have shown in the previous section that we can ensure that the differences introduced by the two keys are integrally canceled with probability greater than $2^{-128}$. In this section, we show how to use this property in order to build a distinguisher and recover the encryption key with a related-key attack. We consider a scenario in which the attacker is given the right to ask for the encryption of plaintexts under the secret encryption key and under a second key which is related to the first one by a fixed difference.

In the following, we denote by $a_{i}, i=1 \cdots 12$ the number of active Sboxes in each round (including the rounds not covered by the characteristic) and by $a_{i \rightarrow j}$ the number of active Sboxes in rounds $i$ to $j$. We provide in Figure 3 a concrete example providing the best trade-off.

\subsection{A First 2R-attack}

We describe here a simple related-key attack on the full cipher and give some optimizations in the following sections. Our attack is based on a differential characteristic (in which all the differences are canceled by the Sboxes) that covers the first 10 rounds and ends with a 2-round key-recovery.

The two points that make the 2-round key-recovery holds are the following: first, the characteristic - that cancels all the differences - allows us to obtain a

good filter on the ciphertexts. Second, a property of the compression function allows us to invert the compression step with a limited complexity. 

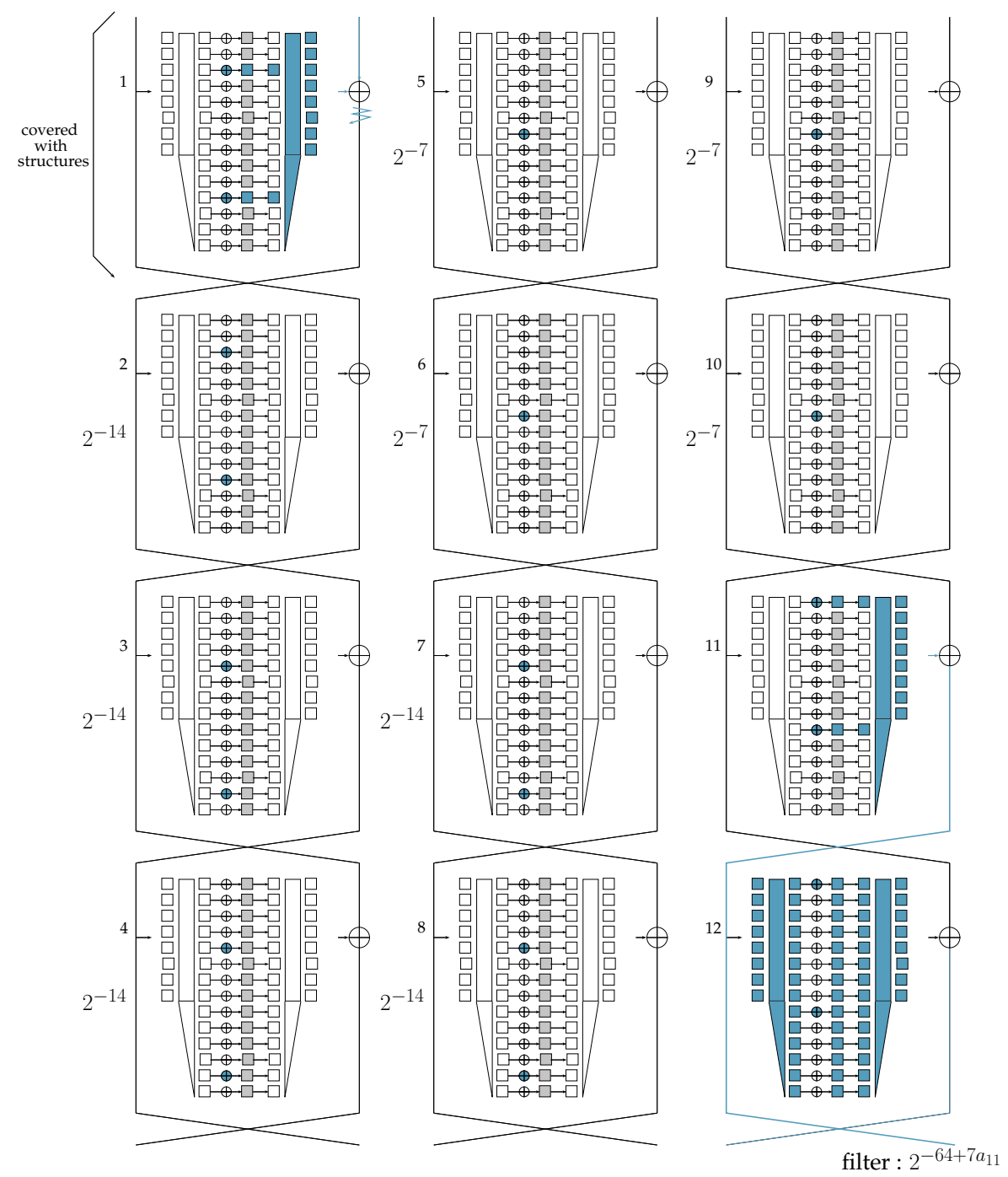

Fig. 3. Related-Key Attack on Full Round PICARO. This figure represents the variant providing the best data complexity: $2^{99}$, with a time complexity of $2^{107.4}$. The master key differences are positioned in bit 16 and 80 .

Ciphertext Filter. For a plaintext and a pair of keys following the characteristic, the state entering round 11 is free of differences, and we know the value of the round-key differences entering the Sboxes at round 11, so we can determine a set of possible differences at the output of round 11. In the worst case scenario, there are $2^{7}$ possible output differences for each active Sbox, so $2^{7 \times a_{11}}$ differences are possible out of $2^{64}$ for the corresponding half of the ciphertext. 
This remark implies that we can filter out some pairs that for sure do not follow the characteristic.

Property of the Compression Function. The following proposition will be extensively used in the attack. It shows that the knowledge of the output of the compression function and of any 6 bytes of the input uniquely determines all input bytes.

Proposition 1. Two distinct 14-byte words having the same image under the compression function coincide on at most five bytes.

Proof. The compression function is defined by

$$
\text { Compr : } \begin{aligned}
G F\left(2^{8}\right)^{14} & \rightarrow G F\left(2^{8}\right)^{8} \\
x & \mapsto x H
\end{aligned}
$$

where $H$ is a $14 \times 8$ matrix over $G F\left(2^{8}\right)$ defined in Section 2.1. Then, the set of all inputs which have the same image under Compr is an affine subspace of $G F\left(2^{8}\right)^{14}$ of dimension 6, which is a coset of the kernel

$$
\mathcal{C}=\operatorname{ker}(\text { Compr })=\{x: x H=0\} .
$$

Therefore, any two elements $x$ and $x^{\prime}$ having the same output under Comp are such that $\left(x+x^{\prime}\right)$ belongs to $\mathcal{C}$. Moreover, $\mathcal{C}$ can also be seen as the linear code of length 14 and dimension 6 over $G F\left(2^{8}\right)$ with parity-check matrix $H^{T}$. Since $H^{T}$ has been chosen by the designers of PICARO as the generator matrix of an MDS code, it also corresponds to the parity-check matrix of an MDS code, implying that the minimum distance of $\mathcal{C}$ is $14-6+1=9$. Now, assume that there exist two distinct inputs $x$ and $x^{\prime}$ which have the same output under Compr and which coincide on 6 input bytes. Then, $\left(x+x^{\prime}\right)$ is a non-zero element of $\mathcal{C}$ and it has at most $14-6=8$ nonzero bytes, which contradicts the fact that the minimum distance of $\mathcal{C}$ equals 9 .

Moreover, for any fixed output $y$ of Compr and any set $I \subset\{1, \ldots, 14\}$ of 6 input positions, the unique 14-byte word equal to a given $\alpha$ on $I$ can be determined by elementary algebra. We first observe that the matrix $H$ defining the compression function is equal to

$$
\left(\begin{array}{l}
\mathrm{ld}_{8} \\
Z^{T}
\end{array}\right)
$$

where $Z$ denotes the $8 \times 6$-right part of matrix $G$ defined in Section 2.1. Then, the set of all words whose image equals $y$ is equal to $\mathcal{C}+\widetilde{y}$ where $\widetilde{y}$ is the 14 byte word equal to $y$ on the first 8 bytes and which vanishes on the 6 other bytes, and $\mathcal{C}=\operatorname{ker}(\mathrm{Compr})$ is the linear space spanned by the rows of matrix $M=\left(Z, \operatorname{Id}_{6}\right)$. Let $M_{I}$ (resp. $\left.M_{\bar{I}}\right)$ denote the columns of $M$ corresponding to $I$ (resp. to $\bar{I}=\{1, \ldots, 14\} \backslash I$ ). The previous proposition shows that $M_{I}$ is nonsingular. Therefore, the unique element $x$ in $\operatorname{Compr}^{-1}(y)$ which is equal to $\alpha$ on $I$ corresponds to the sum of $\widetilde{y}$ and of the element in $\mathcal{C}$ which is equal to $\alpha+\widetilde{y}_{\mid I}$ on $I$. This implies that the value of $x$ on $\bar{I}$ is equal to $\widetilde{y}_{\mid \bar{I}}+\left(\alpha+\widetilde{y}_{\mid I}\right) M_{I}^{-1} M_{\bar{I}}$. 


\section{Obtaining suggestions for the values of the subkey at round 12 .}

1. Choose a master-key difference $\Delta$ with a minimum number of active Sboxes in the first 10 rounds and ask for $2^{7 \times a_{1 \rightarrow 10}}$ triples $\left(P_{i}, C_{i}, C_{i}^{\prime}\right)$ generated from different plaintexts $P_{i}$, where $C_{i}$ and $C_{i}^{\prime}$ are respectively the corresponding ciphertext under the secret master key $K$ and the corresponding ciphertext under the key $K^{\prime}=K \oplus \Delta$.

2. Filter out the triples by looking at the ciphertext differences: $2^{7 \times a_{1 \rightarrow 10}-\left(64-7 \times a_{11}\right)}$ triples remain.

3. Since the internal state entering round 11 is free of difference, the left part of the ciphertext difference is equal to the difference at the output of the compression function at round 12. Given that, guess 6 bytes of the difference entering the compression function and deduce the value of the full 112bit difference entering the compression function at round 12 (this value is uniquely determined as shown by Proposition 1). There are $2^{48}$ possibilities for each triple.

4. Starting from the right half of the ciphertext difference, compute the expansion function and add the difference coming from the key difference to deduce the value of the difference entering the Sboxes at round 12 .

5. With the help of the difference distribution table, check the difference transitions of the 14 Sboxes at round 12. If all the transitions are valid, compute the possible intermediate state values that permit these transitions. In order to estimate the average number of states returned by this procedure, we use that, for any Sbox, the product between the probability that a transition is valid and the number of values following this transition is equal to 1 . For PICARO Sbox $S$ (note that the reasoning would be similar for any Sbox), we consider the number $\delta(a, b)$ of inputs $x$ such that $S(x+a)+S(x)=b$, and the number $\tau$ of valid transitions, i.e., $\tau$ is the number of pairs $(a, b)$ such that $\delta(a, b)>0$. The sum of all (non-zero) entries in the difference table of $S$ equals $2^{2 \times 8}$, i.e., the sum over all valid $(a, b)$ of $\delta(a, b)$ equals $2^{2 \times 8}$. It then follows that

$$
\left(\frac{1}{\tau} \sum_{\text {valid }(a, b)} \delta(a, b)\right) \times\left(\frac{\tau}{2^{2 \times 8}}\right)=1 .
$$

Therefore, for each Sbox transition considered in the attack, we obtain in average one intermediate state which satisfies this transition. So, we get a total of

$$
2^{7 \times a_{1 \rightarrow 10}-\left(64-7 \times a_{11}\right)} \times 2^{48}=2^{7 \times a_{1 \rightarrow 11}-16} \text { candidates. }
$$

6. From the ciphertext value, deduce the intermediate state value before the key addition in round 12 . From that, we obtain $2^{7 \times a_{1 \rightarrow 11}-16}$ candidate values for the subkey of round 12 .

This algorithm requires $2^{7 \times a_{1 \rightarrow 10}+1}$ full encryptions for obtaining the initial triples, then $2^{7 \times a_{1 \rightarrow 11}-16+1} \times \frac{1}{12}$ encryptions (since only round 12 is computed) 
to obtain candidates for $k^{12}$, which leads to an overall time complexity corresponding to the cost of

$$
2^{7 \times a_{1 \rightarrow 10}+1}+2^{7 \times a_{1 \rightarrow 11}-18.58} \text { encryptions. }
$$

\subsection{Optimizations}

Reducing the Initial Number of Encryptions. A potential bottleneck in the previous algorithm comes from the data complexity and then from the cost of the generation of the initial data. In this section, we detail a technique (close to the notion of structures which is commonly used in differential attacks) that reduces it by a factor of $2^{7 \times a_{1}}$. The idea here is to let the first round-key difference spread freely and to cancel this difference by introducing a difference in another plaintext.

For each plaintext $P_{i}$ encrypted under the master key $K$, we ask for the encryption of a set of plaintexts $P_{i}^{\text {set }}$ encrypted under the second key. These plaintexts are made by adding to $P_{i}$ all the possible differences coming from the subkey difference. To compute these differences, we exhaust all the $\left(2^{8 \times a_{1}}\right)$ possibilities for the difference at the output of the active Sboxes at round 1 and apply the (linear) compression function.

Since this set contains all the possible differences, one of them corresponds to the actual difference and will cancel the first round difference. Now, we extend that remark by asking the oracle for the encryption of the same set $P_{i}^{\text {set }}$ under the master key. We then possesses $2^{8 \times a_{1}+1}$ plaintexts and each plaintext in the first set is such that the difference introduced by the first round-key is canceled by exactly one plaintext in the second set. To sum up, we have exactly $2^{8 \times a_{1}}$ pairs that lead to a zero difference at the end of round 1 from $2^{8 \times a_{1}+1}$ encryptions only. The other parts in the algorithm remain the same. So to obtain one pair that leads to a zero difference at round 10 we need

$$
2^{7 \times a_{2 \rightarrow 10}-8 \times a_{1}+8 \times a_{1}+1}=2^{7 \times a_{2} \rightarrow 10+1} \text { encryptions. }
$$

If we apply this technique to the previous algorithm, the number of initial encryptions is reduced from $2^{7 \times a_{1 \rightarrow 10}+1}$ to $2^{7 \times a_{2} \rightarrow 10+1}$.

Speeding Up the Master-Key Recovery. The previous algorithm returns candidates for the 112 bits of the subkey at round 12. From that point, we can naively perform an exhaustive search for the remaining 16 bits of the master key, which would lead to an attack with data complexity $2^{7 \times a_{2 \rightarrow 10}+1}$, time complexity $2^{7 \times a_{2 \rightarrow 10}+1}+2^{7 \times a_{1 \rightarrow 11}-16} \times 2^{16}$ and with memory complexity $2^{8 \times a_{1}+1}$ (necessary to store the initial 'structures').

We can further reduce the second term in the time complexity by using the previous rounds to filter out wrong candidates faster than with a trial encryption. Indeed, we can check if the candidate key gives the right Sbox transitions round after round, and discard a candidate as soon as the Sbox transition is wrong. 
The optimal configuration would be the one for which we can deduce from the candidate value for $k^{12}$ the key bits of $k^{1}$ and $k^{11}$ that enter the active Sboxes at rounds 1 and 11 . In such a case, the attacker can directly (i.e. without any additional key guess) check if the candidate value for $k^{12}$ leads to the right Sbox transitions at rounds 1 and 11 . This corresponds to a filter of about $2^{-7 a_{1}}$ and $2^{-7 a_{11}}$. The remaining operations (checking the Sbox transitions at the other rounds and guessing the remaining bits) are comparatively of negligible time complexity.

In case only a few key bits are known, the attacker can also reduce the time complexity by doing this gradual check. For the $2^{7 a_{1 \rightarrow 11}-16}$ pairs and candidate values for $k^{12}$, she can compute the expansion function at the round in which the largest number of information bits is known. At this round, she needs to guess the unknown bits in order to check the active Sbox transitions and then, for the right guesses, she can compute the entire round function in order to access another round. The full round function is then computed only for the guesses leading to the valid Sbox transitions. This occurs with probability $2^{-7}$ while at most 8 bits must be guessed. Therefore, we can consider that this step has negligible time complexity compared to $2^{7 \times a_{1 \rightarrow 11}-18.58}$.

Hence, the total data complexity is $2^{7 \times a_{2 \rightarrow 10}+1}$, the time complexity is reduced to $2^{7 \times a_{2 \rightarrow 10}+1}+2^{7 \times a_{1 \rightarrow 11}-18.58}$, and the memory is unchanged $\left(2^{8 \times a_{1}+1}\right)$.

Choosing $a_{2 \rightarrow 10}$ and $a_{1 \rightarrow 11}$. According to the previous analysis the bottleneck in the time complexity is:

$$
2^{7 \times a_{2 \rightarrow 10}+1}+2^{7 \times a_{1 \rightarrow 11}-18.58} .
$$

It is then parameterized by $a_{2 \rightarrow 10}$ (the amount of active Sboxes from round 2 to 10) and by $a_{1 \rightarrow 11}$ (the amount of active Sboxes from round 1 to 11).

A simple search returns that the minimum for $a_{2 \rightarrow 10}$ is 14 and that the minimum for $a_{1 \rightarrow 11}$ is 17 . Unfortunately, the two minima cannot be reached simultaneously, which gives raise to the following two possible options.

Variant 1: minimizing $a_{2 \rightarrow 10}$. We consider here situations for which $a_{2 \rightarrow 10}=$ 14, the minimum for $a_{1 \rightarrow 11}$ in these cases is 18 (see Table 2 for the difference positions that reach these minima and Figure 3 for an example of related-key attack using this variant).

One of the advantages of this variant is that some of the options we can choose for the master-key difference allow us to speed up the search for the master-key. Indeed, two active Sbox transitions at round 1 and two other ones at round 11 can be checked without any additional key guess, as previously explained. However, this speed-up is imperceptible since it does not decrease the time complexity bottleneck. The final time complexity is

$$
2^{7 \times a_{2 \rightarrow 10}+1}+2^{7 \times a_{1 \rightarrow 11}-18.58}=2^{7 \times 14+1}+2^{7 \times 18-18.58}=2^{99}+2^{107.4}=2^{107.4} .
$$

The total data complexity is $2^{7 \times a_{2 \rightarrow 10}+1}=2^{99}$, and the memory complexity is $2^{8 \times a_{1}+1}=2^{17}$. 
Table 2. List of all master-key differences (of weight less than 4) minimizing $a_{2 \rightarrow 10}$ $\left(a_{2 \rightarrow 10}=14\right)$ with the smallest value for $a_{1 \rightarrow 11}\left(a_{1 \rightarrow 11}=18\right)$. The last 2 columns indicate the number of key bytes (and bits) deduced from $k^{12}$ at the positions corresponding to the active Sboxes at rounds 1 and 11.

\begin{tabular}{|c|c|c|c|c|c|c|c|}
\hline diff & positions & $\| a_{1}$ & $a_{2 \rightarrow 10}$ & $a_{11}$ & $a_{1 \rightarrow 11}$ & known bytes (bits) in $k_{1}$ & known bytes (bits) in $k_{11}$ \\
\hline 11 & 107 & 2 & 14 & 2 & 18 & $1(14)$ & $2(16)$ \\
\hline 12 & 108 & 2 & 14 & 2 & 18 & $1(14)$ & $2(16)$ \\
\hline 13 & 109 & 2 & 14 & 2 & 18 & $1(14)$ & $2(16)$ \\
\hline 14 & 110 & 2 & 14 & 2 & 18 & $1(14)$ & $2(16)$ \\
\hline 15 & 111 & 2 & 14 & 2 & 18 & $1(14)$ & $2(16)$ \\
\hline 16 & 80 & 2 & 14 & 2 & 18 & $2(16)$ & $2(16)$ \\
\hline 17 & 81 & 2 & 14 & 2 & 18 & $2(16)$ & $2(16)$ \\
\hline 18 & 82 & 2 & 14 & 2 & 18 & $2(16)$ & $2(16)$ \\
\hline 19 & 83 & 2 & 14 & 2 & 18 & $2(16)$ & $0(14)$ \\
\hline 20 & 84 & 2 & 14 & 2 & 18 & $2(16)$ & $0(14)$ \\
\hline 21 & 85 & 2 & 14 & 2 & 18 & $2(16)$ & $0(14)$ \\
\hline 22 & 86 & 2 & 14 & 2 & 18 & $2(16)$ & $0(14)$ \\
\hline 23 & 87 & 2 & 14 & 2 & 18 & $2(16)$ & $0(14)$ \\
\hline 24 & 88 & 2 & 14 & 2 & 18 & $0(4)$ & $0(14)$ \\
\hline 25 & 89 & 2 & 14 & 2 & 18 & $0(4)$ & $0(14)$ \\
\hline 26 & 90 & 2 & 14 & 2 & 18 & $0(4)$ & $0(14)$ \\
\hline 27 & 91 & 2 & 14 & 2 & 18 & $0(4)$ & $0(0)$ \\
\hline 28 & 92 & 2 & 14 & 2 & 18 & $0(4)$ & $0(0)$ \\
\hline 29 & 93 & 2 & 14 & 2 & 18 & $0(4)$ & $0(0)$ \\
\hline 30 & 94 & 2 & 14 & 2 & 18 & $0(4)$ & $0(0)$ \\
\hline 31 & 95 & 2 & 14 & 2 & 18 & $0(4)$ & $0(0)$ \\
\hline 32 & 96 & 2 & 14 & 2 & 18 & $0(0)$ & $0(0)$ \\
\hline 33 & 97 & 2 & 14 & 2 & 18 & $0(0)$ & $0(0)$ \\
\hline 34 & 98 & 2 & 14 & 2 & 18 & $0(0)$ & $0(0)$ \\
\hline 35 & 99 & 2 & 14 & 2 & 18 & $0(0)$ & $0(2)$ \\
\hline 36 & 100 & 2 & 14 & 2 & 18 & $0(0)$ & $0(2)$ \\
\hline 37 & 101 & 2 & 14 & 2 & 18 & $0(0)$ & $0(2)$ \\
\hline 38 & 102 & 2 & 14 & 2 & 18 & $0(0)$ & $0(2)$ \\
\hline 39 & 103 & 2 & 14 & 2 & 18 & $0(0)$ & $0(2)$ \\
\hline 40 & 104 & 2 & 14 & 2 & 18 & $0(12)$ & $0(2)$ \\
\hline 41 & 105 & 2 & 14 & 2 & 18 & $0(12)$ & $0(2)$ \\
\hline
\end{tabular}


Variant 2: minimizing $a_{1 \rightarrow 11}$. If we choose to minimize $a_{1 \rightarrow 11}$, the minimum value of $a_{2 \rightarrow 10}$ is 15 , and the time necessary to generate the data becomes $2^{7 \times a_{2 \rightarrow 10}+1}=2^{106}$.

An exhaustive search among these configurations, as presented in Table 3 . shows that none of them allow to do the direct sieving with $k^{1}$ and $k^{11}$ that was possible in the previous variant. The attack obtained when the master-key difference is chosen at positions 27 and 123 is depicted in Figure 4. The final data complexity (which is also the time complexity bottleneck) is $2^{7 \times 15+1}=2^{106}$, the time complexity is $2^{7 \times a_{2 \rightarrow 10}+1}+2^{7 \times a_{1 \rightarrow 11}-18.58}=2^{106}+2^{100.4}=2^{106}$ and the memory complexity is $2^{8 \times a_{1}+1}=2^{9}$.

Table 3. List of all master-key differences (of weight less than 4) minimizing $a_{1 \rightarrow 11}$ $\left(a_{1 \rightarrow 11}=17\right)$ with the smallest value for $a_{2 \rightarrow 10}\left(a_{2 \rightarrow 10}=15\right)$. The last 2 columns indicate the number of key bytes (and bits) deduced from $k^{12}$ at the positions corresponding to the active Sboxes at rounds 1 and 11 .

\begin{tabular}{|ll||c|c|c|c|c|c|}
\hline \multicolumn{2}{|l|}{ diff. positions } & $a_{1}$ & $a_{2 \rightarrow 10}$ & $a_{11}$ & $a_{1 \rightarrow 11}$ & known bytes (bits) in $k_{1}$ & known bytes (bits) in $k_{11}$ \\
\hline \hline 27 & 123 & 1 & 15 & 1 & 17 & $0(2)$ & $0(0)$ \\
\hline 28 & 124 & 1 & 15 & 1 & 17 & $0(2)$ & $0(0)$ \\
\hline 29 & 125 & 1 & 15 & 1 & 17 & $0(2)$ & $0(0)$ \\
\hline 30 & 126 & 1 & 15 & 1 & 17 & $0(2)$ & $0(0)$ \\
\hline 91 & 123 & 1 & 15 & 1 & 17 & $0(2)$ & $0(0)$ \\
\hline 92 & 124 & 1 & 15 & 1 & 17 & $0(2)$ & $0(0)$ \\
\hline 93 & 125 & 1 & 15 & 1 & 17 & $0(2)$ & $0(0)$ \\
\hline 94 & 126 & 1 & 15 & 1 & 17 & $0(2)$ & $0(0)$ \\
\hline
\end{tabular}

\section{Conclusion}

In this paper we exhibit related-key attacks on the full-round block cipher PICARO. Our attacks exploit a weakness in the key-schedule as well as the nonbijectivity of the Sbox. We think that a stronger key-schedule with a better diffusion would help thwarting this type of attack.

Despite the fact that our related-key attacks do not represent a threat to the cipher in other more realistic scenarios, the authors aimed at providing relatedkey resistance, and we believe that such claim should be revised. 

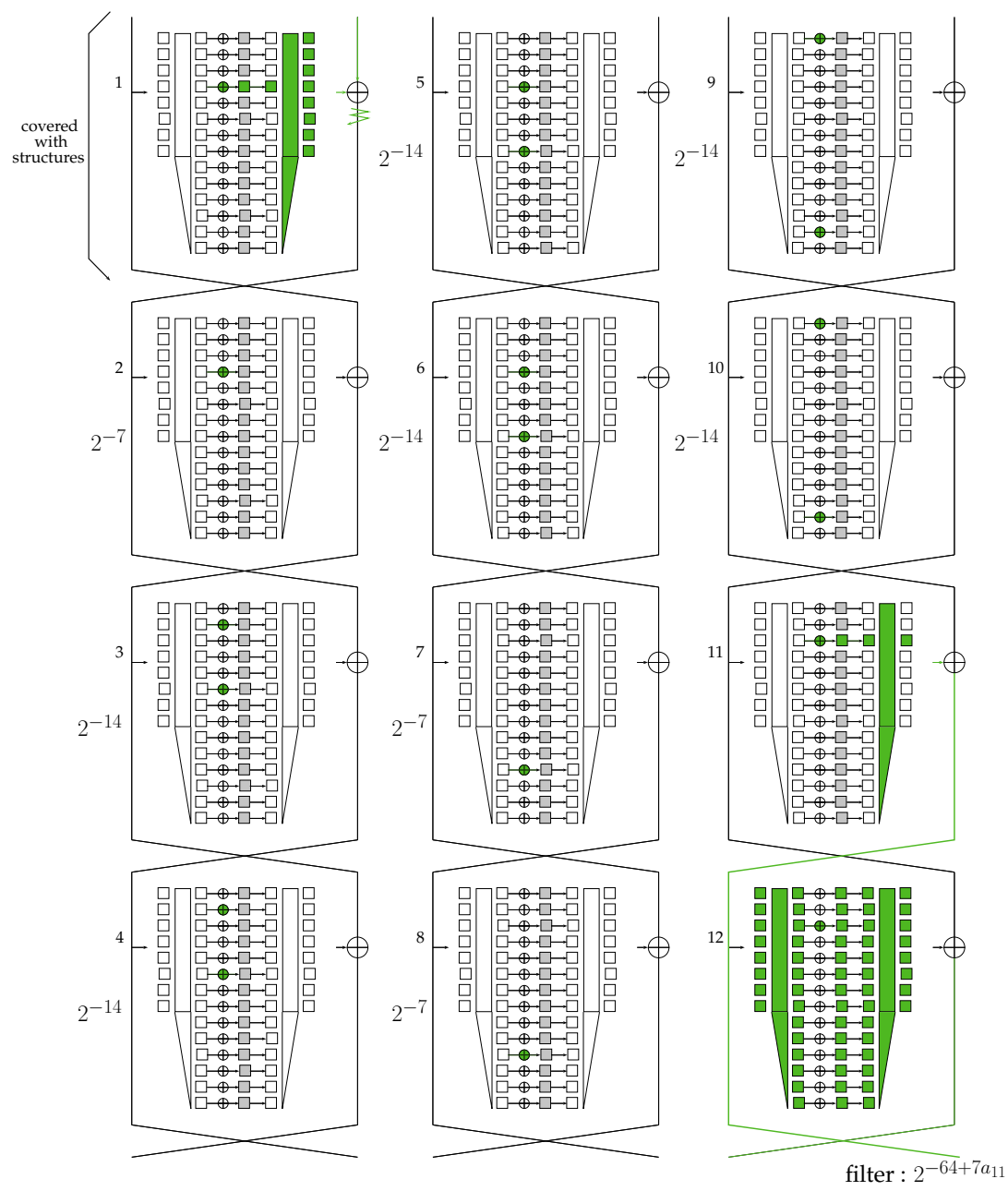

Fig. 4. Related-Key attack on full-round PICARO . This figure represents the attack with the best time complexity: $2^{106}$, with a data complexity of $2^{106}$. The master-key differences are located at bits 27 and 123 .

\section{References}

1. Biham, E.: New Types of Cryptanalytic Attacks Using Related Keys. Journal of Cryptology 7(4), 229-246 (1994)

2. Biham, E., Shamir, A.: Differential Cryptanalysis of DES-like Cryptosystems. Journal of Cryptology 4(1), 3-72 (1991)

3. Biryukov, A., Wagner, D.: Slide Attacks. In: Fast Software Encryption - FSE'99. Lecture Notes in Computer Science, vol. 1636, pp. 245-259. Springer (1999)

4. Canteaut, A., Chabaud, F.: A New Algorithm for Finding Minimum-Weight Words in a Linear Code: Application to McEliece's Cryptosystem and to Narrow-Sense 
BCH Codes of Length 511. IEEE Transactions on Information Theory 44(1), 367378 (1998)

5. Carlet, C.: Relating Three Nonlinearity Parameters of Vectorial Functions and Building APN functions from Bent Functions. Designs, Codes and Cryptography 59(1-3), 89-109 (2011)

6. Gérard, B., Grosso, V., Naya-Plasencia, M., Standaert, F.X.: Block Ciphers That Are Easier to Mask: How Far Can We Go? In: Cryptographic Hardware and Embedded Systems - CHES 2013. Lecture Notes in Computer Science, vol. 8086, pp. 383-399. Springer (2013)

7. Grosso, V., Leurent, G., Standaert, F., Varici, K.: LS-Designs: Bitslice Encryption for Efficient Masked Software Implementations. In: Fast Software Encryption - FSE 2014. Lecture Notes in Computer Science, vol. 8540, pp. 18-37. Springer (2014)

8. Piret, G., Roche, T., Carlet, C.: PICARO - A Block Cipher Allowing Efficient Higher-Order Side-Channel Resistance. In: Applied Cryptography and Network Security - ACNS 2012. Lecture Notes in Computer Science, vol. 7341, pp. 311-328. Springer (2012)

9. Piret, G., Roche, T., Carlet, C.: PICARO - A Block Cipher Allowing Efficient Higher-Order Side-Channel Resistance - extended version -. IACR Cryptology ePrint Archive 2012, 358 (2012), http://eprint.iacr.org/2012/358

10. Rivain, M., Prouff, E.: Provably Secure Higher-Order Masking of AES. In: Cryptographic Hardware and Embedded Systems - CHES 2010. Lecture Notes in Computer Science, vol. 6225, pp. 413-427. Springer (2010)

\section{A PICARO Sbox}

\begin{tabular}{|c|c|c|c|c|c|c|c|c|c|c|c|c|c|c|c|c|}
\hline & & & 02 & & & & & & & & & & & & & \\
\hline 0 & 08 & $0 \mathrm{c}$ & ש & 6 & 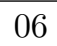 & 04 & 05 & 06 & 5 & 04 & $0 \mathrm{c}$ & 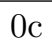 & 04 & 03 & 05 & 03 \\
\hline 0 & $0 \mathrm{a}$ & $1 f$ & 29 & $\mathrm{~b}$ & $4 \mathrm{~b}$ & 5 & 62 & $7 \mathrm{~b}$ & 82 & 95 & af & bf & $\mathrm{c} 5$ & $\mathrm{~d} 9$ & ? & \\
\hline 0 & 01 & $2 \mathrm{~d}$ & 45 & $d$ & $8 \mathrm{a}$ & $c$ & $\mathrm{Cl}$ & ea & $f$ & $\mathrm{C}$ & $\mathrm{d}$ & $1 \mathrm{du}$ & $1 \mathrm{C}$ & 35 & $5 \mathrm{f}$ & 75 \\
\hline 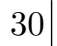 & Of & 34 & 61 & 52 & $c 2$ & $\mathrm{fb}$ & 3 & 92 & 13 & $2 b$ & 74 & 4 & $\mathrm{db}$ & e1 & b3 & \\
\hline 40 & 01 & 44 & 81 & $\mathrm{C}$ & 92 & UD & 13 & 52 & b3 & 10 & 34 & 14 & D & 61 & $d J$ & \\
\hline 50 & $0 \mathrm{e}$ & 59 & $\mathrm{a} 4$ & f8 & $\mathrm{d} 8$ & 87 & 8 & 28 & 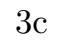 & 7 & 09 & с9 & 7 & 4 & 1s & \\
\hline 60 & 02 & 63 & $\mathrm{ca}$ & $\mathrm{da}$ & $1 \mathrm{~d}$ & 71 & $\mathrm{~d} 7$ & $\mathrm{bd}$ & 27 & 41 & e3 & 83 & 31 & $5 \mathrm{a}$ & $\mathrm{f} 7$ & \\
\hline 70 & Of & 74 & e1 & 92 & 52 & $2 \mathrm{~b}$ & b3 & ?) & 3 & la & 4 & 34 & $\mathrm{fb}$ & 1 & 12 & \\
\hline 80 & 02 & 83 & $9 a$ & $1 d$ & $\mathrm{bd}$ & 31 & 27 & $\mathrm{ad}$ & $\mathrm{f} 7$ & 71 & 63 & e3 & 41 & ca & $\mathrm{d} 7$ & \\
\hline ח & $0 \mathrm{e}$ & 99 & b4 & 28 & f8 & 67 & $4 c$ & $\mathrm{~d} 8$ & $7 \mathrm{c}$ & 7 & c9 & 59 & 87 & 4 & $3 c$ & \\
\hline $\mathrm{a} 0$ & $0 \mathrm{a}$ & af & $\mathrm{d} 9$ & $7 \mathrm{~b}$ & $3 b$ & 95 & $\mathrm{e} 2$ & $4 \mathrm{~b}$ & 62 & $\mathrm{c} 5$ & bf & 1f & 55 & f9 & 82 & \\
\hline 100 & $0 \mathrm{a}$ & bf & f9 & $4 \mathrm{~b}$ & $7 \mathrm{~b}$ & c5 & 82 & $3 \mathrm{~b}$ & 2 & 55 & $1 \mathrm{f}$ & af & 95 & 9 & & \\
\hline $\mathrm{c} 0$ & $0 \mathrm{e}$ & c9 & 14 & $\mathrm{~d} 8$ & 28 & e7 & $3 c$ & f8 & $4 c$ & 87 & 59 & 99 & 67 & $\mathrm{a} 4$ & $7 \mathrm{c}$ & \\
\hline Qu & 01 & $\mathrm{dd}$ & 35 & ea & $6 a$ & $D C$ & 01 & $8 a$ & $\mathrm{CI}$ & IC & 10 & $2 \mathrm{a}$ & $\mathrm{dc}$ & 10 & II & 40 \\
\hline e0 & 02 & e3 & $5 a$ & $\mathrm{bd}$ & ad & 41 & $\mathrm{f} 7$ & $1 d$ & $\mathrm{~d} 7$ & 31 & 83 & 63 & 71 & $\mathrm{a}$ & 27 & \\
\hline & 01 & $\mathrm{fd}$ & 75 & & & & 91 & $6 a$ & $\pi 1$ & $\mathrm{dc}$ & $2 d$ & & $D C$ & 45 & $\mathrm{Cl}$ & \\
\hline
\end{tabular}

